\title{
Influence of the turning process on the distortion of disks for gear manufacture
}

\author{
Tim Kohlhoff · Jens Sölter · Ekkard Brinksmeier
}

Received: 31 May 2011 / Accepted: 14 July 2011 / Published online: 30 July 2011

(C) The Author(s) 2011. This article is published with open access at Springerlink.com

\begin{abstract}
This study presents investigations on the fundamental mechanisms of part distortion during the machining of disks (cylindrical gear base bodies). The work focuses on the effects of machining induced residual stresses and inhomogeneous material removal. In this context the workpiece clamping was investigated as a major impact factor on the distortion. In order to analyze and describe the complex shape deviations, an appropriate distortion characterization method was developed. Since the distortion of disks directly affects the gear wheel quality, the results will help to understand the formation of gear distortion as well as to reduce and compensate it.
\end{abstract}

Keywords Distortion - Manufacturing · Disk - Gear · Clamping $\cdot$ Residual stress

\section{List of symbols}

$\begin{array}{ll}a_{k}(\mathrm{~mm}) & \text { Amplitude } \\ a_{p}(\mathrm{~mm}) & \text { Depth of cut } \\ C & \text { Constant } \\ d(\mathrm{~mm}) & \text { Distance from centerline } \\ d_{a}, d_{i}(\mathrm{~mm}) & \text { Outer, inner diameter } \\ f(\mathrm{~mm}) & \text { Feed rate } \\ F_{c, c j}(\mathrm{~N}) & \text { Centrifugal force, acting on clamping jaws } \\ F_{c l}(\mathrm{~N}) & \text { Clamping force } \\ F_{c l d}(\mathrm{~N}) & \text { Dynamic clamping force } \\ F_{c l s}(\mathrm{~N}) & \text { Static clamping force } \\ h(\mathrm{~mm}) & \text { Height } \\ L(\mathrm{~mm}) & \text { Length }\end{array}$

T. Kohlhoff $(\square) \cdot$ J. Sölter · E. Brinksmeier Manufacturing Technologies, Foundation Institute of Material Science (IWT), Badgasteiner Str. 3, 28359 Bremen, Germany e-mail: kohlhoff@iwt.uni-bremen.de

URL: www.iwt-bremen.de

$\begin{array}{ll}m_{c j}(\mathrm{~kg}) & \text { Mass of clamping jaws } \\ r(\mathrm{~mm}) & \text { Radius } \\ r_{c j}, r_{t}(\mathrm{~mm}) & \text { Radial position of clamping jaw, tool } \\ R^{2} & \text { Coefficient of determination } \\ U(\mu \mathrm{m}) & \text { Measurement uncertainty } \\ v_{c}(\mathrm{~m} / \mathrm{min}) & \text { Cutting speed } \\ \sigma_{t}\left(\mathrm{~N} / \mathrm{mm}^{2}\right) & \text { Tangential surface stress } \\ \varphi\left({ }^{\circ}\right) & \text { Circumference angle } \\ \text { RS } & \text { Residual stress } \\ \text { SRA } & \text { Stress relief annealing } \\ \text { SJ } & \text { Segment jaw } \\ \text { GM1 } & \text { Geometry measurement before final cut } \\ \text { GM2 } & \text { Geometry measurement after final cut } \\ \text { GM3 } & \text { Geometry measurement after final SRA } \\ \text { FFT } & \text { Fast Fourier transform }\end{array}$

\section{Introduction}

For the manufacture of power transmission parts like cylindrical gears, the surface layer is hardened after machining to increase the wear resistance. This heat treatment leads to undesired changes of size and shape, the so called distortion. The resulting deviations have to be removed by a second machining process, mostly grinding, to ensure the required part tolerances. As abrasive processes are relatively expensive and time consuming, they are critical for a cost-effective manufacture. Distortion is not only caused by the heat treatment process. As different studies show, a so called distortion-potential is induced into the workpiece along the entire process chain. The distortion-potential is released during the heat treatment which results in distortion [1, 6-8]. 
During turning, mechanical and thermal loads cause a plastic deformation of the surface layer generating residual stress (RS) in the whole workpiece [2-4]. Residual stresses and their variations are always linked to distortion, depending on their dimensions and the workpiece cross section [4]. A heat treatment (stress relief annealing, hardening) changes the residual stress state which leads to changed equilibrium conditions, accompanied by a deformation of the workpiece [5-8]. For this reason, residual stresses are valuated as a carrier of distortion-potential [9].

Figure 1 gives an overview on the influence of machining on distortion and distortion-potential of rings. An inhomogeneous material removal and the generation of residual stresses mainly affect the distortion and distortionpotential of rings. Both mechanisms are influenced by the workpiece clamping and the chip formation process.

For example, rotation-symmetric workpieces are often clamped with three-jaw-chucks, which are cost-effective and useful to adapt to different part diameters [10]. However these clamping devices can cause high elastic deformations of the workpiece because of high clamping pressures between ring and clamping jaws [11, 12]. This leads to an inhomogeneous material removal as the effective cutting depth (which locally differs from the adjusted cutting depth) increases at the positions of the clamping jaws. After machining, the clamped ring has a perfectly round inner surface but has an alternating wall thickness along the circumference. The release of the clamping forces causes a deformation to a noncircular inner surface. Additionally elastic workpiece deformation depends on the process forces which can be controlled by the cutting parameters, especially the depth of cut $a_{p}$ and the feed rate $f$.

Furthermore the clamping influences the residual stress state [13]. Sölter [11] explains the generation of periodical residual stresses by superposition of residual stresses from machining (source stress) with bending stresses due to the elastic ring deformation. The clamping force $F_{c l}$ causes elastic bending stresses (dashed line in Fig. 2). During machining the yield stress is exceeded so that after machining the clamped ring shows constant surface stresses (dotted line). Unclamping the ring leads to an inversion

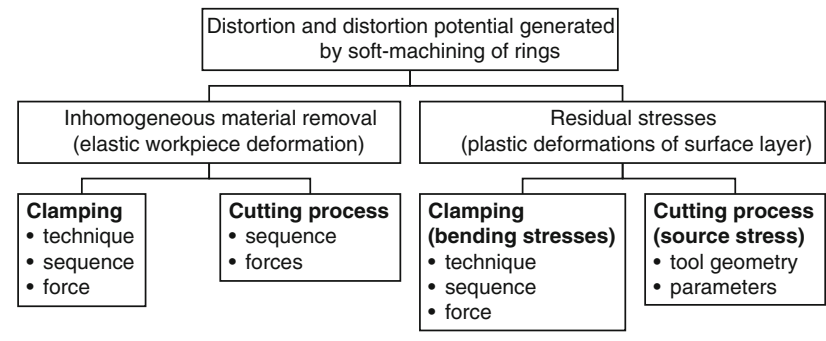

Fig. 1 Influence of machining on distortion and distortion-potential of rings

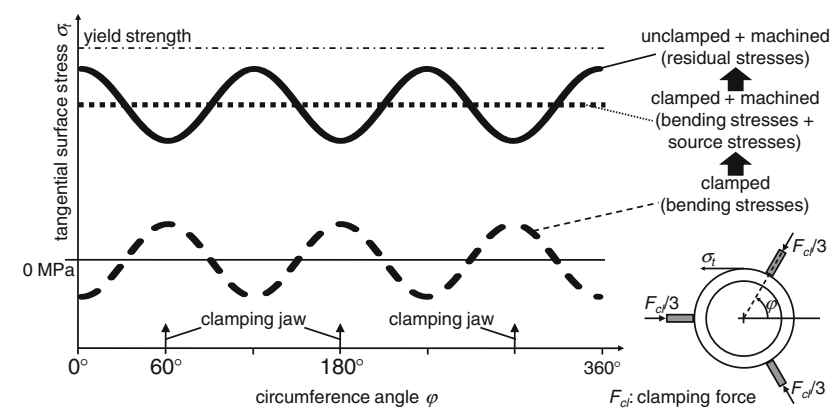

Fig. 2 Model of generation of periodic residual stresses during machining of rings (according to [11])

of the periodic bending stresses. As a result periodic residual stresses occur (continuous line).

\section{Objectives and procedure}

The aim of this study was to reveal the fundamental mechanisms of distortion generation in turning of disks with a centered hole. The resulting shape deviation and distortion were measured and characterized. Due to the complex distortion behavior of the disks, an appropriate characterization method had to be developed.

For the experiments forged disks with a height of about $h \approx 21 \mathrm{~mm}$ were used, made of 20MnCr5 (SAE 5120) steel and annealed to a ferrite-pearlite structure. After machining, the workpiece had an outer diameter of $d_{a}=120 \mathrm{~mm}$ and a bore diameter of $d_{i}=45^{\mathrm{H} 7} \mathrm{~mm}$. A comparatively small height of $4 \mathrm{~mm}$ was realized to ensure a detectability of small effects.

For the experimental examination of disk distortion a special procedure of experiment and measurements was designed (Fig. 3). In a first step the disks were machined on all faces except for the last cut on the bottom face and were stress relief annealed afterwards. By this way an equal, almost residual stress-free state of all disks could be ensured. The shape and size were subsequently determined in a first geometry measurement (GM1). In the following last cut, machining parameters were varied according to the experimental matrix shown in Table 2. Afterwards a second geometry measurement (GM2) was carried out. The disks were stress relief annealed a second time to release the induced residual stresses of the last cut and were measured a last time (GM3).

The machining process was carried out on a vertical NCturning center Index V250 using cooling lubricant. Cemented carbide inserts CNMG 1204 08-PM and CNMG 1606 08-PM were used as cutting tools, which had a multilayercoating $\left(\mathrm{Al}_{2} \mathrm{O}_{3}, \mathrm{TiN}, \mathrm{TiCN}\right)$. In premachining the forged green body, which had an average height of $h \approx 21 \mathrm{~mm}$, was turned to a height of $h=5 \mathrm{~mm}$ (Table 1). 


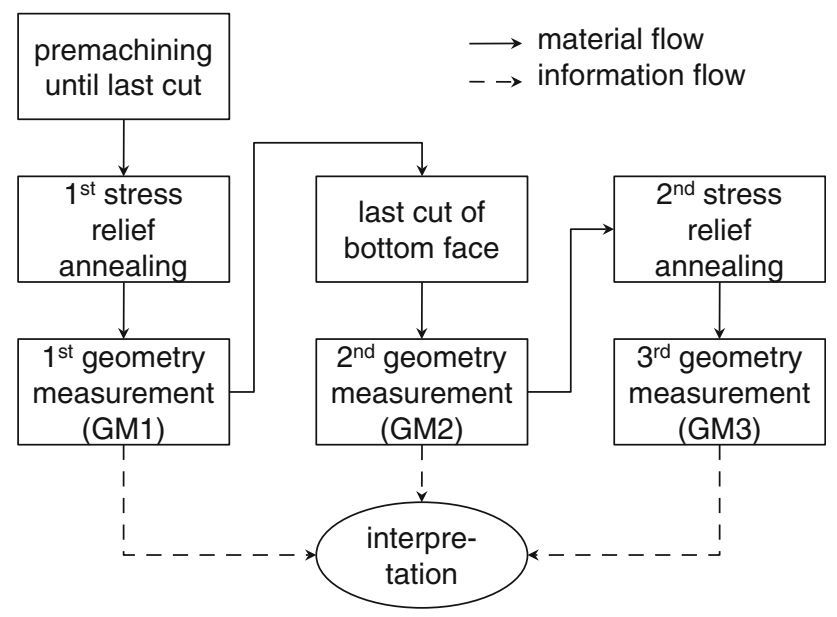

Fig. 3 Procedure of experiment and analysis

Table 1 Parameters of premachining process

\begin{tabular}{lllll}
\hline Process sequence & $\begin{array}{l}\text { Number } \\
\text { of cuts }\end{array}$ & $\begin{array}{l}\text { Cutting } \\
\text { depth } \\
a_{p} / \mathrm{mm}\end{array}$ & $\begin{array}{l}\text { Feed } \\
\text { rate } \\
f / \mathrm{mm}\end{array}$ & $\begin{array}{l}\text { Cutting } \\
\text { speed } \\
v_{c} / \mathrm{m} / \mathrm{min}\end{array}$ \\
\hline 1. Face turning top side & 1 & 1 & 0.3 & 250 \\
2. External turning & 1 & 1 & 0.2 & 250 \\
3. Boring & 1 & 1 & 0.3 & 250 \\
4. Face turning bottom & 15 & 1 & 0.3 & 250 \\
\hline
\end{tabular}

In the first part of premachining, the green body was adjusted by a form-locking clamping device ("Inogrip" of HWR Spanntechnik GmbH). Except for the bottom face, the disk was machined completely to a height of $h=20 \mathrm{~mm}$ (step 1-3 of Table 1). For machining of the bottom face and for the final process variations, the workpieces were externally clamped with segment jaws (SJ). For an analysis of the effects of the clamping it is essential to be able to reproduce the orientation of the disk in the chuck. Therefore all disks were marked at an indexing position $\left(\varphi=0^{\circ}\right)$ and were adjusted in the same orientation in the clamping device.

During the final cut, four variations (two factors on two levels each) with five repetitions each were carried out (Table 2). Thus the validity of achieved results for a larger process window was ensured. As a standard process condition a depth of cut of $a_{p}=1 \mathrm{~mm}$ and a feed rate of $f=0.3 \mathrm{~mm}$ was defined. The selection of the parameter variations provided a full factorial $2^{2}$ designed experiment.

During stress relief annealing (SRA), the specimens were slowly heated up with $5^{\circ} \mathrm{C}$ per minute until they reached an annealing temperature of $650^{\circ} \mathrm{C}$. This temperature was kept constant for an hour and afterwards the disks were cooled down to room temperature in the switched off oven.
Table 2 Experimental matrix

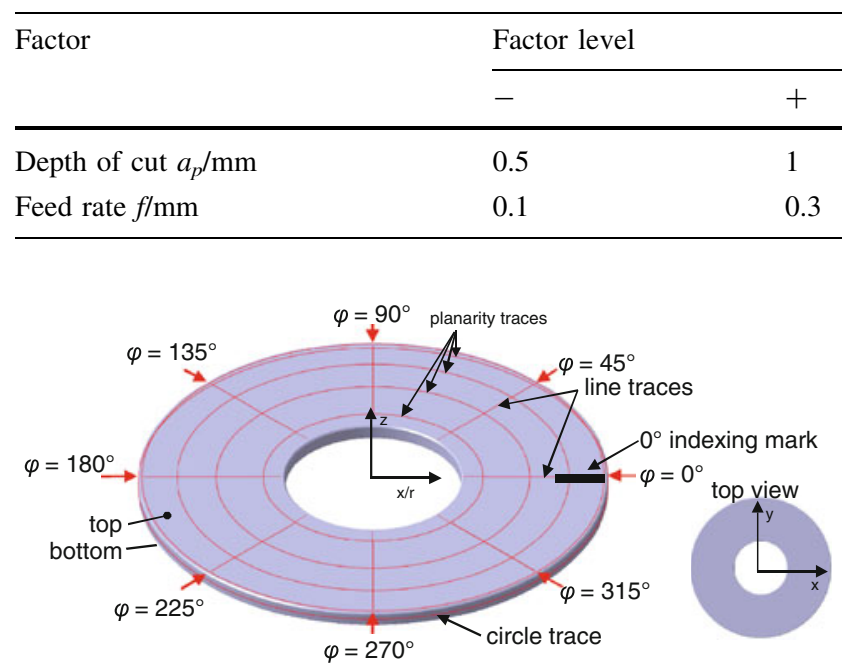

Fig. 4 Measurement strategy

Table 3 Conclusions of differences of measurement values

\begin{tabular}{ll}
\hline Differences & Geometry changes due to \\
\hline GM2 - GM3 & Residual stresses \\
GM3 - GM1 & Inhomogeneous material removal \\
GM1 - GM2 & $\begin{array}{c}\text { Residual stresses and inhomogeneous } \\
\text { material removal }\end{array}$ \\
\hline
\end{tabular}

GM geometry measurement

The geometry measurements were carried out on a coordinate measuring machine Mahr PRIMAR MX4. According to the manufacturer, the one-, two-, and threedimensional length measurement uncertainty $(U)$ is: $U_{1}=1.2 \mu \mathrm{m}+L / 500 \mu \mathrm{m}, \quad U_{2}=1.5 \mu \mathrm{m}+L / 300 \mu \mathrm{m}$, $U_{3}=2 \mu \mathrm{m}+L / 300 \mu \mathrm{m}$ with $L$ as the length of the measuring trace. The shape of the disk was captured by a mesh of measuring traces using the Scanning-Method (Fig. 4). To be able to correlate deviations with their possible causes in machining, all measuring traces were referenced by setting the angle of the indexing mark to $\varphi=0^{\circ}$. Eight line traces were used to measure the radial shape. The four plane-measuring traces were performed at radii of 28,40 , 50 and $59 \mathrm{~mm}$. The circularity-measuring trace was carried out in a height of $2 \mathrm{~mm}$. The entire geometry measurement took place in a single clamping setup of each disk. Therefore in all following diagrams of top face data the material is located in negative $z$-direction whereas for bottom face data it is located in positive $z$-direction.

In industrial applications, an evaluation of the process deviations is often based on measurements of the finished part, only (GM2). However for the distortion analysis, geometry changes between the different states are of main importance. A correlation of geometry changes to their causes is summarized in Table 3. 


\section{Methods}

In contrast to the commonly used shape characteristics like planarity and out-of-roundness, the examination of the fundamental mechanisms leading to distortion requires a more detailed analysis. At first, the shape deviations in radial direction (evaluated from the line traces) and those in tangential direction (evaluated from the planarity traces) were analyzed separately.

\subsection{Radial shape deviations}

To describe the radial shape deviation, a number of five workpieces of each variation provided eight line traces on both top and bottom. Therefore the results on the radial shape deviations presented in this study are based on average values of 160 line traces of top and bottom face.

Line traces of each disk face varied with respect to their axial position ( $z$-coordinate) due to shape deviations along the circumferential direction. Therefore the mean line trace was calculated and the mean $z$-coordinate of the resulting curve was set to $z=0$. As a consequence the line traces of each coordinate measurement could be compared directly, even if the thicknesses of the compared disk-states differ. However no conclusion about the change of height could be given anymore.

From the resulting curve, the slope was determined by the differentiation of the approximated equation of the regression line. Bending of the line trace was computed by approximating a second order polynomial and differentiating twice with respect to the radius.

\subsection{Tangential shape deviations}

From the planarity traces measured in absolute Cartesian coordinates, an interpolation plane was calculated. Therefore all measuring points were described by position vectors and a plane in Hessian normal form was set up. The interpolation plane was determined by minimizing the distance of all measuring points to the plane using the least square method. This was computed iterative by means of the "MS Excel Solver", which uses the normal unit vector and the distance of the plane to the point of origin as variables. These processed planarity traces allow a characterization of the tangential shape deviations.

The highest deviations appeared at the outer measuring radius of $59 \mathrm{~mm}$. So the major deviations (which represent the planarity) were only evaluated for this specific radius. In general, the planarity of the disks can have the same value while showing different shape deviations (Fig. 5).

Therefore, for a precise characterization and evaluation of the shape deviation, the measuring data were separated into their harmonics using a Fourier analysis. Amplitudes

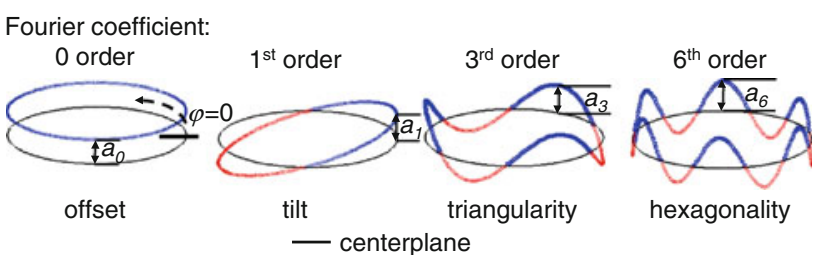

Fig. 5 Different characteristics of tangential measurements with same planarity

$a_{k}$ and angles of the different orders made it possible to correlate the resulting shape deviations with their physical causes (Fig. 5). The phase angle determines the positions of maximal and minimal planar deviations from the reference plane with respect to the indexing mark $\left(\varphi=0^{\circ}\right)$.

\section{Results and discussion}

In the following the results of all three geometry measurements and changes in state (cf. Table 3 ) are presented. The results of all four variations were qualitatively the same. Thus only results from the standard process will be quantitatively analyzed in the following.

\subsection{Radial shape deviation}

Figure 6 compares the arithmetical means of the line traces of all three geometry measurements. It has to be considered that the trace line is plotted against the radius. So, for an impression of the cross sectional shape, the graph has to be mirrored at the ordinate.

The results of the first geometry measurement (GM1) show a concave shape (a so called "dishing") of the top face as well as of the bottom face which is indicated by the directions of the slopes. The origin of this shape deviation can only be an inhomogeneous material removal since the disks are in a stress relieved state. Measurements of the face shape in a clamped condition showed that the clamping force bends the disk out of the clamping jaws. Obviously more material is removed at lower radii than at higher ones as the positive/negative slope at the top/bottom face indicate. The smaller slope of the top face results from the higher moment of inertia the disk had during machining $\left(h_{\text {top }} \approx 21 \mathrm{~mm}\right.$ vs. $\left.h_{\text {bottom }}=5 \mathrm{~mm}\right)$ which leads to less deformation by the clamping force.

Furthermore, the bottom face shows a negative bending which is caused by a decrease of the dynamic clamping force $F_{c l}$ along the feed travel. As the face sides of the disks were turned with a constant cutting speed, the spindle revolution speed had to be increased along the feed travel towards the disk center. This leads to a reduction of the preset static clamping force $F_{c l s}$ due to the rising centrifugal 

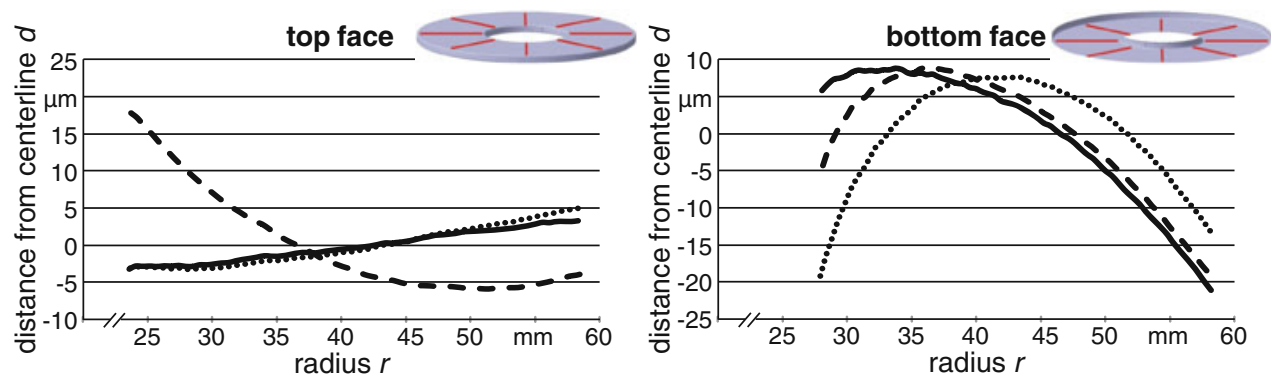

\begin{tabular}{|c|c|c|c|}
\cline { 2 - 4 } \multicolumn{1}{c|}{} & $\begin{array}{c}\text { slope / } \\
\mu \mathrm{m} / \mathrm{mm}\end{array}$ & $\begin{array}{c}\text { bending / } \\
1 / 1000\end{array}$ & $\begin{array}{c}\text { straightness } \\
/ \mu \mathrm{m}\end{array}$ \\
\hline $\mathrm{GM} 1-$ & 0.20 & 0.00 & 6.5 \\
\hline $\mathrm{GM} 2--\cdot$ & -0.60 & 0.08 & 12.0 \\
\hline GM3 $\cdots \cdots \cdot \cdot \cdot \cdot$ & 0.30 & 0.00 & 8.5 \\
\hline
\end{tabular}

\begin{tabular}{|l|c|c|c|}
\cline { 2 - 4 } \multicolumn{1}{c|}{} & $\begin{array}{c}\text { slope / } \\
\mu \mathrm{m} / \mathrm{mm}\end{array}$ & $\begin{array}{c}\text { bending / } \\
1 / 1000\end{array}$ & $\begin{array}{c}\text { straightness } \\
/ \mu \mathrm{m}\end{array}$ \\
\hline GM1- & -0.90 & -0.09 & 30.5 \\
\hline GM2- - & -0.70 & -0.13 & 28.0 \\
\hline GM3-...... & -0.03 & -0.20 & 27.0 \\
\hline
\end{tabular}

GM: geometry measurement

Fig. 6 Arithmetical means of line traces

force that acts on the clamping jaws. So the elastic deformation of the disk is reduced along the feed travel, thus less material is removed near the center. This effect did not show up at the top face as in this state the disk had a lower sensitivity to the clamping force due to its height.

The second geometry measurement (GM2) represents the disk shape in a stress loaded state. The top face shows a considerable change compared to GM1. It bends upwards at the center respectively the outer edge tilts downwards which is a dishing in the opposite direction. As in the final cut material was removed from the bottom face only, this effect had to be caused by machining induced residual stresses. This hypothesis is proven by the results of the third geometry measurement (GM3) of the top face. After the second stress relief annealing the top face deforms back into its initial shape (GM1).

At the bottom face the superposition of residual stresses and inhomogeneous material removal affects the radial shape deviation (GM2). A concave shape deviation is generated by the second stress relief annealing (GM3). In order to separate the influence of residual stress from the inhomogeneous material removal, shape changes had to be evaluated (Fig. 7).

At the top face only residual stresses influence the shape leading to considerable shape changes between GM2 and GM3. Therefore the shape change at the bottom face is the same for GM2-3. Regarding the differences in the stated straightness and slope, it has to be considered that on the top face the measurement of the line traces began at a smaller radius than on the bottom face. A localization of the analyzing area to $28 \mathrm{~mm}<r<58 \mathrm{~mm}$ would lead to equal results for straightness and slope. The direction of bending indicates an impact of compressive residual stresses.

GM3-1 characterizes the inhomogeneous material removal caused by the final cut. High negative values for low radii indicate a strong decrease of the effective depth of cut, leading to a positive slope. It is assumed that this is caused by decreasing clamping forces with decreasing radial positions of the tool.

The dynamic clamping force $F_{c l d}$ is calculated by

$F_{c l d}=F_{c l s}-F_{c, c j}$

with $F_{c l s}$ as static clamping force and $F_{c, c j}$ as centrifugal force, acting on the clamping jaws. As the cutting speed is kept constant along the feed travel, the revolution of the spindle rises with a decreasing of the radial tool position $r_{t}$. Therefore $F_{c, c j}$ is a function of $r_{t}$ :

$F_{c, c j}\left(r_{t}\right)=m_{c j} \cdot r_{c j} \cdot \frac{v_{c}^{2}}{r_{t}^{2}}$

with $m_{c j}$ as mass of the clamping jaws and $r_{c j}$ as radial position of clamping jaws. As the inhomogeneous material removal depends on the dynamic clamping force it is proportional to the function $-r_{t}^{(-2)} \cdot C$ with $C$ as a constant which is indicated by a high value of the coefficient of determination $R^{2}$ with $R^{2}=0.97$ (Fig. 8).

The inhomogeneous material removal caused a change of the shape contrary to the effect of the machining induced residual stresses as shown by the direction of slope and bending. This circumstance was not obvious from the isolated results of each state (cf. Fig. 6) because of the superposition of these two effects.

In practical applications, the accuracy of the machining process often is evaluated by the workpiece geometry after the process (GM2). In this case the shape of the top face would lead to an adjustment of the process even though its shape deviation resulted from the turning of the bottom face. This procedure leads to the desired result of accurate workpiece geometry. Though, for a comprehensive understanding of the causes of distortion, in terms of Distortion Engineering, this approach is not useful. Here, the knowledge of the whole machining sequence is essential. 

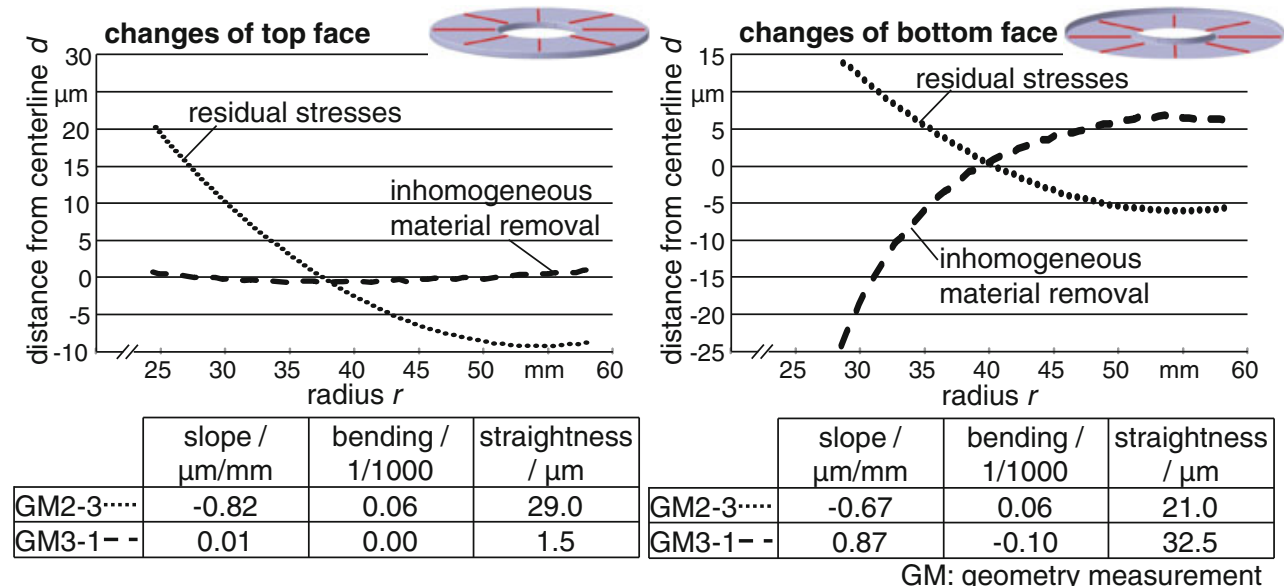

Fig. 7 Radial changes by residual stresses (GM2-3) and inhomogeneous material removal (GM3-1)

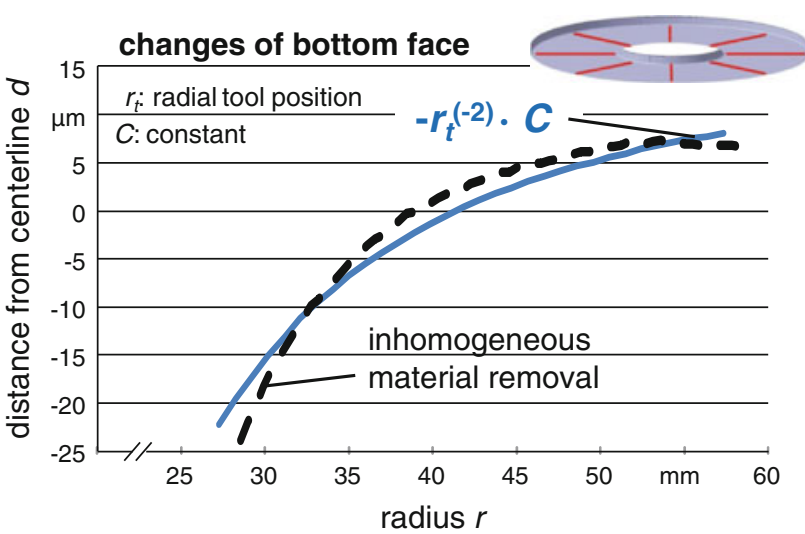

Fig. 8 Comparison of the inhomogeneous material removal with decrease of clamping force (continuous line is proportional to $F_{c l}$ )

Especially a consideration of shape changes rather than absolute shapes helps to distinguish effects of inhomogeneous material removal and those of residual stresses.

\subsection{Tangential shape deviation}

The tangential shape deviations show a qualitative similar progression for all radii but the deviations increase for higher radii. Therefore the effects can be analyzed best by the data of the outer trace $(r=59 \mathrm{~mm})$. The averaged planarity traces of this radius are plotted against the circumference angle in Fig. 9. As many effects of the disk deformation can be traced back to clamping, the position of the segment jaws (SJ) is indicated in the diagrams. A fast Fourier transform (FFT) of the measured data showed meaningful results for 3rd and 6th order. So these values are presented in terms of amplitude and phase angle in tables below the diagrams.

The tangential shape of the initial state (GM1) shows deviations of $3 \mathrm{rd}$ and 6th order. These deviations result from inhomogeneous material removal during premachining. Similar to the results of the radial shape in this state (cf. Fig. 6) the deviations of the bottom face are more

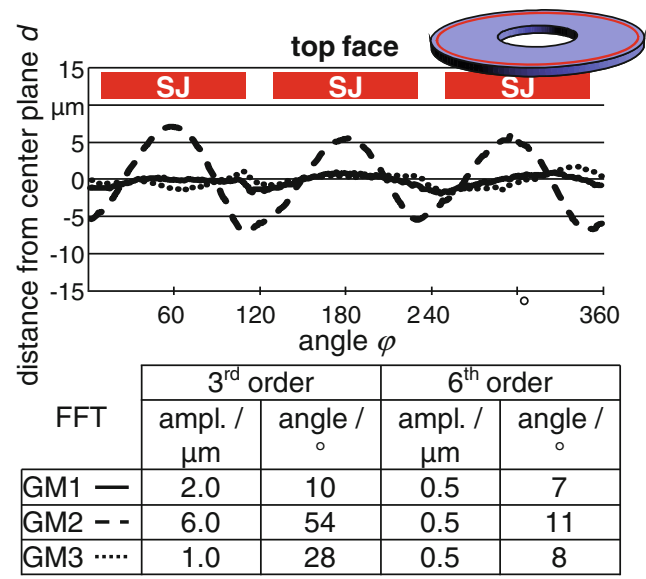

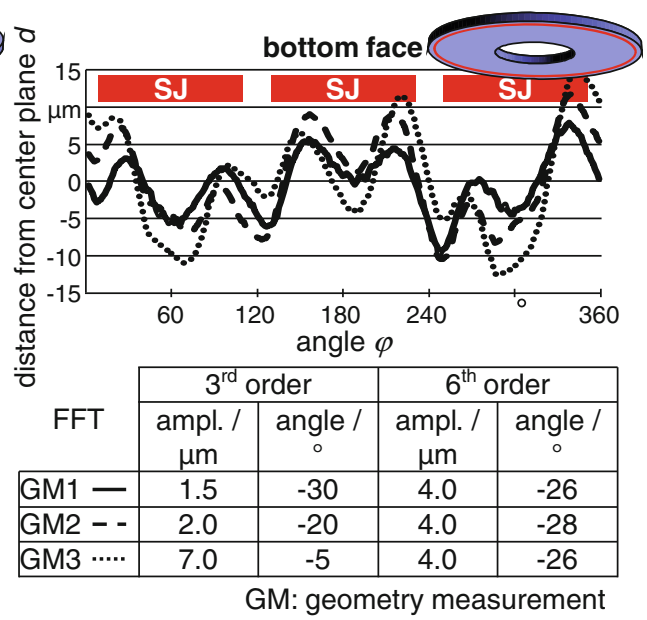

Fig. 9 Averaged planarity traces at $r=59 \mathrm{~mm}$ 


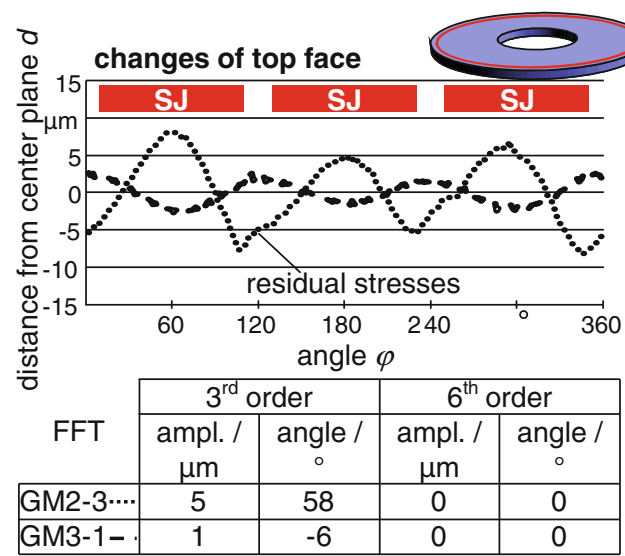

Fig. 10 Changes of tangential shape deviations by residual stresses (GM2-3) and inhomogeneous material removal (GM3-1)

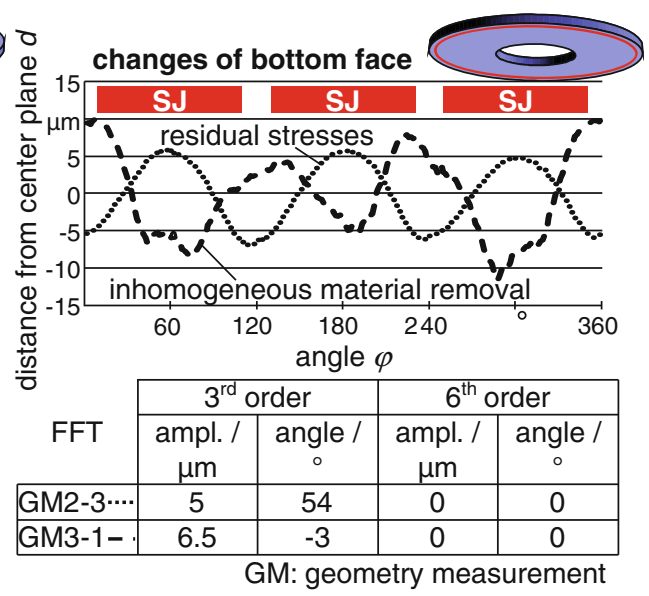

distinctive than of top face. During machining the disk is deformed by the clamping forces, which leads to an inhomogeneous material removal. Due to the smaller height during machining of the bottom face, the disk deformation due to clamping is higher. Thus the remaining deviations exceed those of the top face.

A deviation of 6th order was generated in the premachining process. Values of 6th order of all three states (GM1, GM2, GM3) do not change. Therefore the occurrence of this 6th order deviation was not considered in this study.

The tangential shape of the top face after the final cut (GM2) shows a significant deviation of 3rd order. As no material on the top face was machined this deviation had to be caused by the residual stresses that were induced during machining of the bottom face. This hypothesis was proven by the results of GM3 as this deviation vanished after SRA.

In general the effects of inhomogeneous material removal and residual stresses are much harder to identify from the results of the bottom shape. This is because of the high amplitudes of 6th order which hides the effects, and because of the superposition of the effects themselves. Therefore an investigation of the shape changes is necessary (Fig. 10).

The tangential shape changes due to residual stresses (GM2-3) of top and bottom face are the same. They result from a 3rd order progression of the residual stresses. The phase angle of approx. $60^{\circ}$ correlates to the position of the segment jaws. The generation of the $3 \mathrm{rd}$ order residual stresses can be explained by the same mechanism that occurs during the machining of rings (cf. Fig. 2), namely the superposition of bending stresses from clamping with source stresses due to cutting.

A closer look on the comparison of 3rd order deviation of GM3 of top and bottom identifies a crucial difference (cf. Fig. 9). As the residual stresses cause an advancement of bottom face planarity, the planarity of the top face is worsen by the same effect.

The changes of the bottom face due to an inhomogeneous material removal (GM3-1) proof the impact of the clamping device. The phase angle of approx. $0^{\circ}$ indicates that during machining the material was pushed out of the chuck in the areas between the segment jaws. This is because the turned diameter of the chuck never fits the diameter of the disks precisely. In this case a smaller diameter of the segment jaws seams to lead to six contact areas where the clamping forces act on the disk (Fig. 11).

On the top face the results of GM3-1 show a small change of 3rd order of the radial shape. As no material was removed from this face, this change has to be a measurement artifact. The results of GM1 and GM3 are slightly shifted regarding the 3 rd order $\left(18^{\circ}\right)$. This causes a 3 rd order deviation by subtraction of the values of these two states. As the workpieces are manually loaded into the measuring machine, a variation of the orientation between two measurements is always given. Even though the same disk in the same state would be measured twice, a small twist would lead to the calculation of nonexistent shape changes (Fig. 12). Moreover, the orientation of the change, represented by the phase angle, does not correlate to the position of the segment jaws and therefore is understood as an error.

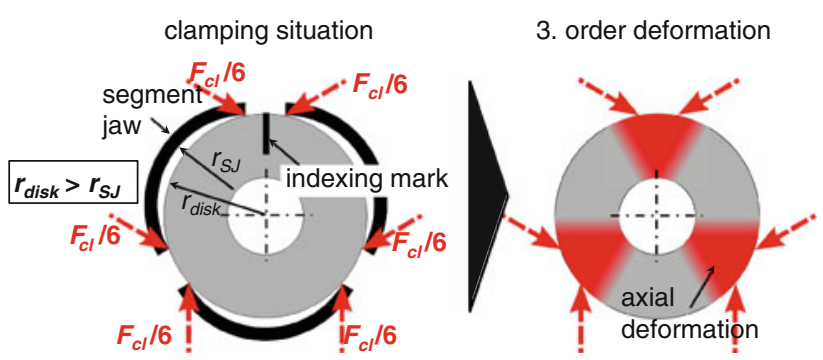

Fig. 11 Elastic deformation due to clamping forces $F_{c l}$ 

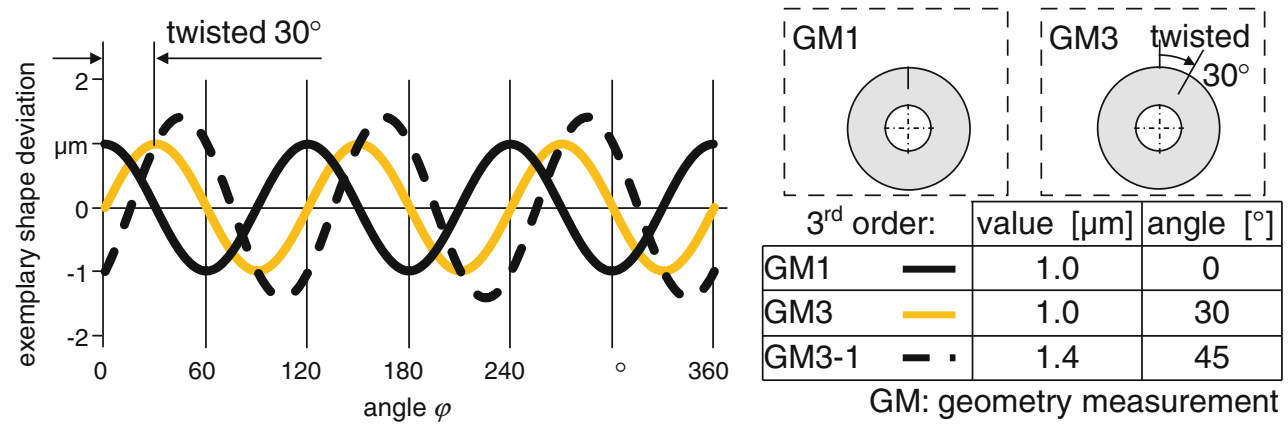

Fig. 12 Model of the generation of a nonexistent shape change by measurement process

In general the fast Fourier transform turned out as an effective method for the analysis of tangential shape deviations. In addition to FFT an analysis of the shape changes should be performed. Thus negative effects that superimpose the results of the analyzed step can be eliminated (in this case deviations of 6th order of the premachining process). Nevertheless this method is sensitive to inaccuracies during the measurement. Therefore the results of FFT should not be used without being put into perspective.

\section{Conclusions}

Machining induced distortion of disks leads to bended, sloped and/or corrugated surfaces which are dissimilar for top and bottom face. In order to be able to analyze this complex geometry with regard to the source of the distortion, the radial and tangential shapes were investigated separately in this study. Besides an analysis of the disk geometry after every manufacturing step, an identification of distortion mechanisms requires an investigation of the changes between different states. These presented approaches allow detecting the cause of distortion and shape deviations due to the turning of disks. As disks can be seen as a base body of cylindrical gears, a direct impact of disk distortion on gears is obvious. In long term the results shall help finding proper strategies for the prevention and compensation of distortion during the machining of gear wheels.

Acknowledgments This work was carried out within the „Sonderforschungsbereich 570, Distortion Engineering“. The authors would like to thank the Deutsche Forschungsgemeinschaft (DFG) for the financial support.

Open Access This article is distributed under the terms of the Creative Commons Attribution Noncommercial License which permits any noncommercial use, distribution, and reproduction in any medium, provided the original author(s) and source are credited.

\section{References}

1. Hoffmann F, Keßler O, Lübben Th, Mayr P (2002) „Distortion Engineering “-Verzugsbeherrschung in der Fertigung. HärtereiTechnische Mitteilungen 57(3):213-217

2. Tönshoff HK (1966) Eigenspannungen und plastische Verformungen im Werkstück durch spanende Bearbeitung. Dissertation, Technische Hochschule Hannover

3. Denkena B, Boehnke D, de León L (2008) Machining induced residual stress in structural aluminum parts. Prod Eng Res Devel 2(3):247-253

4. Brinksmeier E, Cammett JT, König W, Leskovar P, Peters J, Tönshoff HK (1982) Residual Stress-Measurement and causes in machining processes. CIRP Ann Manuf Technol 34(1): 485-490

5. Volkmuth J (1996) Eigenspannungen und Verzug. HärtereiTechnische Mitteilungen 51(3):145-154

6. Volkmuth J, Sjöblom U, Slycke J, Thuvander A (2000) Effect of uneven residual stresses on dimensional changes and variations of through hardening bearing steel rings. In: Procedures of the 20th ASM heat treating society conference, USA, pp 455-460

7. Thuvander A (2002) Out of roundness distortion of bearing rings owing to internal stresses from tube bending. Mater Sci Technol $18: 312-318$

8. Volkmuth J, Lane S, Jung M, Sjöblom U (2005) Einfluss ungleichmäßiger Eigenspannungen in Wälzringen vor dem Härten auf Formabweichungen nach dem Härten. Härterei-Technische Mitteilungen 60(6):317-322

9. Nowag L, Sölter J, Walter A, Brinksmeier E (2006) Effect of machining parameters and clamping technique on residual stresses and distortion of bearing rings. Materialwissenschaft und Werkstofftechnik 37(1):45-51

10. Grote C, Brinksmeier E, Garbrecht M (2009) Distortion Engineering in turning processes with standard clamping systems. Materialwissenschaft und Werkstofftechnik 40(5-6):385-389

11. Sölter J (2010) Ursachen und Wirkmechanismen der Entstehung von Verzug infolge spanender Bearbeitung. Dissertation, Universität Bremen, Shaker Verlag, Aachen

12. Brinksmeier E, Sölter J, Grote C (2007) Distortion engineeringidentification of causes for dimensional and form deviations of bearing rings. CIRP Ann Manuf Technol 56(1):109-112

13. Hirsch Th (2003) Entwicklung von Eigenspannungszuständen im Fertigungsprozess. Härterei-Technische Mitteilungen 58(3): $110-126$ 